\title{
Avaliação sensorial e físico-química de sorvete com baixo teor de lactose e adição de polpa de abacaxi (Ananas comosus L. Merril)
}

\author{
Sensorial and physico chemical evaluation of low lactose ice cream added with \\ pineapple pulp (Ananas comosus L. Merril)
}

\author{
Sandra Mari Guedes ${ }^{1}$ \\ José Raniere Mazile Vidal Bezerra² \\ Ângela Moraes Teixeira ${ }^{3}$ \\ Maurício $\operatorname{Rigo}^{4}(*)$
}

\section{Resumo}

Visando atender a população intolerante a lactose com um produto de boa qualidade nutricional, este artigo apresenta os resultados obtidos da adição de polpa de abacaxi na formulação de sorvete de baixo teor de lactose. O sorvete padrão foi formulado com leite integral de baixo teor de lactose. Três outras formulações de sorvetes foram preparadas com adição de polpa de abacaxi nas concentrações de 10,20 e 30\%, em relação à massa total do sorvete. A avaliação do efeito da substituição do leite pela polpa de abacaxi foi efetuada por teste sensorial e análises físico-químicas. Os resultados mostram que a formulação com adição de $10 \%$ da polpa de abacaxi apresentou maior aceitação sensorial e maiores teores de cinzas e fibras, em relação à amostra padrão.

Palavras-chave: sorvete, sobremesa, aceitação sensorial.

\section{Abstract:}

To attend the lactose intolerant population with a product of good nutritional quality, this paper shows results obtained from pineapple pulp addition in the ice cream formulation with low lactose content. Standard ice cream was formulated with low lactose milk. Three other ice cream formulations were prepared with addition of pineapple pulp in 10,20 and 30\% levels. The effect of pineapple pulp addition in the low lactose ice cream was evaluated by sensorial tests and physico-chemical analysis. The results show that the formulation with the addition of $10 \%$ pineapple pulp showed the highest sensory acceptance and higher ash and fiber contents compared to the standard sample.

Keywords: Sensory acceptance, ice cream, dessert

\footnotetext{
1 Graduação em Engenharia de Alimentos; Vínculo: Colaborador, Enquadramento Funcional: Produção de Alimentos Neutracêuticos na Universidade Estadual do Centro-Oeste, UNICENTRO, Brasil; Endereço: R. Simeão Varela de Sá,03, 85040-080 - Vila Carli, Guarapuava - PR - Brasil; E-mail: sandramarididi@yahoo.com.br

2 Dr.; Engenharia de Alimentos; Professor Associado de Engenharia de Alimentos da Universidade Estadual do Centro Oeste - Guarapuava - Paraná - Brasil; Endereço: R. Simeão Varela de Sá, 03, 85040-080 - Vila Carli, Guarapua va - PR - Brasil; E-mail: raniere@unicentro.br

3 Dra.; Tecnologia de Alimentos; Professora na Universidade Estadual do Centro-Oeste; Endereço: R. Simeão Varela de Sá,03, 85040-080 - Vila Carli, Guarapuava - PR - Brasil; E-mail: amteixeira11@yahoo.com.br

4 Dr.; Engenharia de Alimentos; Professor Associado da Universidade Estadual do Centro-Oeste, UNICENTRO, Brasil; Endereço: R. Simeão Varela de Sá, 03, 85040-080 - Vila Carli, Guarapuava - PR - Brasil; E-mail: mrigo@ unicentro.br $(*)$ Autor para correspondência.
}

\begin{tabular}{llllll}
\hline Ambiência & Guarapuava (PR) & v.14n.1 & p. 01-08 & Jan/Abr. 2018 & ISSN $1808-0251$
\end{tabular}




\section{Introdução}

Segundo a Associação Brasileira das Indústrias e do Setor de Sorvetes o consumo per capita em litros / ano de sorvete no Brasil foi de 6,41 em 2014 e de 5,59 em 2015. A produção de sorvete de massa no Brasil vinha com tendência de crescimento contínua de 502 milhões de litros em 2003 a 923 milhões de litros em 2014, já a produção de sorvete de massa no Brasil em 2015 e 2016 foi de 794 e 675 milhões de litros, respectivamente (ABIS, 2016). A queda de produção de sorvete está relacionada com recessão econômica que aflige o Brasil nos últimos anos.

A Agência Nacional de Vigilância Sanitária (ANVISA) segundo a Portaria n 379, de 26 de abril de 1999 define como gelados comestíveis os produtos alimentícios obtidos a partir de uma emulsão de gorduras e proteínas, com ou sem adição de outros ingredientes e substâncias, ou de uma mistura de água, açúcares e outros ingredientes ou substâncias que tenham sido submetidas ao congelamento, em condições tais que garantam conservação do produto no estado congelado ou parcialmente congelado durante a armazenagem, o transporte e a entrega ao consumo (BRASIL, 1999). Essa também classifica os gelados comestíveis quanto à composição básica e em relação ao processo de fabricação, onde se estabelece a seguinte classificação para sorvetes de massa ou cremosos: são misturas homogêneas ou não de ingredientes alimentares, batidas e resfriadas até o congelamento, resultando em massa aerada.

Segundo Pereda (2007), a estrutura do sorvete é baseada em uma mistura complexa, sendo ela heterogênea, mas ao mesmo tempo emulsão, gel, suspensão e espuma, da qual a ligação é mantida devido ao congelamento. O sorvete é uma espuma na qual as bolhas de ar estão cobertas por cristais de gelo, glóbulos de gorduras individualizados e cristais de lactose. As bolhas de ar são muito importantes, pois tornam o sorvete mais leve, tornam o produto macio e deformável a mastigação e atuam como isolantes do frio intenso.

A intolerância a lactose acomete indivíduos que possuem insuficiência na produção da enzima responsável pela quebra do açúcar do leite - a lactose (LONGO, 2006). A utilização de leites com baixo teor de lactose ou derivados de soja e arroz são uma das melhores alternativas para a população intolerante a lactose, por esta razão se torna de extrema importância o desenvolvimento de novos produtos que não agridam a saúde destes indivíduos, abrindo novas escolhas de produtos alimentícios. A importância do desenvolvimento de produtos com baixa lactose se deve ao fato de que os alimentos que são derivados do leite, como queijo, iogurtes e o próprio leite causam alguns sintomas desconfortáveis, gases, náuseas, diarreias, inchaços e outros, em pessoas que possuem intolerância a lactose, essas pessoas representam um total de $70 \%$ dos brasileiros adultos (FAEDO, 2013).

O abacaxi tem sua origem da América Central e dentre as frutas tropicais é uma das mais populares no mundo. Suas características são favoráveis à saúde, já que ele não é uma fruta considerada calórica, e possui quantidades de vitaminas A, B e C elevadas, bem como sais minerais, carboidratos e fibras (SILVA, 1996).

Segundo Queiroz e Treptow (2006) a aceitação e a preferência são conceitos distintos, sendo que a preferência é a expressão do mais alto grau de gostar e a aceitação é a experiência caracterizada por uma atitude positiva, é o fato de um indivíduo ou população ser favorável ao consumo de um produto. 
A expectativa gerada por um produto influi na aceitabilidade e intenção de compra e de maneira geral, um produto gera dois tipos de expectativas, a sensorial e a hedônica. A sensorial se caracteriza pela conviç̧ão do consumidor de que o produto apresenta determinadas características sensoriais que podem influenciar sua opinião ao consumir o alimento e a hedônica, o consumidor crê gostar do produto. $\mathrm{Na}$ expectativa hedônica estão intrinsecamente ligados os conceitos de satisfação e insatisfação, que podem ser medidos como a diferença entre o esperado e o percebido (QUEIROZ; TREPTOW, 2006).

De acordo com a NBR 12994 (ABNT,1994) os métodos subjetivos/afetivos são classificados em comparação pareada, ordenação, escala hedônica e escala de atitude.

Visando agregar valor ao sorvete com baixo teor de lactose, os objetivos deste estudo foram: elaborar e determinar a composição centesimal da polpa de abacaxi (Ananas comosus L. Merril); desenvolver e avaliar as propriedades físico-químicas de formulações sorvetes com substituição parcial do leite por polpa de abacaxi e realizar testes sensoriais, buscando conhecer a aceitação do produto.

\section{Materiais e métodos}

\section{Local de pesquisa}

Este trabalho foi realizado no Departamento de Engenharia de Alimentos da Universidade Estadual do Centro-Oeste, nos laboratórios de Análise de Alimentos, Processos na Indústria de Alimentos e Analise Sensorial.

Obtenção da matéria prima

Todos os ingredientes foram comprados em estabelecimentos comerciais de Guarapuava, PR. O leite empregado neste trabalho foi o longa vida integral com baixo teor de baixo lactose. Os abacaxis (Ananas comosus L. Merril) utilizados neste experimento foram comprados e selecionados de um único lote.

Obtenção da polpa de abacaxi

Os abacaxis foram higienizados em água corrente e mergulhados em solução de hipoclorito de sódio na concentração de $10 \mathrm{mg} / \mathrm{L}$, por 10 minutos. Após a lavagem foram descascados e processados em despolpadeira com tela de 1,6 $\mathrm{mm}$ de abertura, envasados em embalagem plástica (PVC) e armazenados em freezer a $-18^{\circ} \mathrm{C}$.

Formulações das misturas

A elaboração da mistura dos ingredientes dos sorvetes foi desenvolvida a partir de testes preliminares, resultando nas composições apresentadas na Tabela 1. Foi elaborada uma formulação padrão (FP) contendo de leite integral com baixo teor de lactose e outras com substituição parcial do leite por $10 \%$, 20 \% e $30 \%$ de polpa de abacaxi, denominadas de F1, F2 e F3, respectivamente. 
Tabela 1: Formulações dos sorvetes com diferentes proporções de polpa de abacaxi.

\begin{tabular}{lcccc}
\hline \multicolumn{1}{c}{ Ingredientes } & FP (\%) & F1 (\%) & F2 (\%) & F3 (\%) \\
\hline Leite de baixa lactose & 72,46 & 62,46 & 52,46 & 42,46 \\
Sacarose & 26,04 & 26,04 & 26,04 & 26,04 \\
Liga neutra & 0,72 & 0,72 & 0,72 & 0,72 \\
Emulsificante neutro & 0,72 & 0,72 & 0,72 & 0,72 \\
Goma xantana & 0,04 & 0,04 & 0,04 & 0,04 \\
Polpa de abacaxi & 0 & 10 & 20 & 30 \\
Total & 100 & 100 & 100 & 100 \\
\hline
\end{tabular}

FP-Formulação padrãosemadição depolpa;F1-Formulaçãocom 10\% depolpa;F2-Formulaçãocom 20\%depolpa;F3-Formulação com $30 \%$ de polpa.

Preparo do sorvete

A elaboração das formulações de sorvete foi realizada através do seguinte procedimento: 1. Pesagem e mistura dos ingredientes leite e sacarose (mistura). 2. Pasteurização da mistura a $63{ }^{\circ} \mathrm{C}$ por 30 minutos. 3. Processo de maturação da mistura por 4 horas a $1{ }^{\circ} \mathrm{C}$. 4 . Adição da liga neutra, polpa de abacaxi, emulsificante neutro e goma xantana à mistura em batedeira até completa homogeneização. 5. Armazenamento do sorvete em potes plásticos, a temperatura de $-18^{\circ} \mathrm{C}$, até o momento das análises.

Avaliação Sensorial

Considerando-se o interesse e disponibilidade 60 provadores não treinados da comunidade acadêmica da Universidade Estadual do Centro-Oeste participaram do teste de aceitação, todos com idade entre 18 e 50 anos sendo $70 \%$ do sexo feminino. Todos os participantes assinaram o Termo de Consentimento Livre e Esclarecido antes da sessão sensorial.

A formulação padrão (FP) e as três formulações F1, F2 e F3 com adição de polpa de abacaxi foram avaliadas quanto à aparência, textura, aroma, sabor, cor e aceitação global utilizando-se escala hedônica estruturada de nove pontos, cujos extremos correspondem a gostei muitíssimo (9) e desgostei muitíssimo (1). As amostras foram oferecidas em copos plásticos, codificadas com números de três dígitos aleatórios, acompanhadas de um copo de água para realização do branco entre as amostras. As formulações foram oferecidas aos julgadores de forma monódica sequencial (DUTCOSKY, 1996).

Avaliou-se a intenção de compra, utilizando-se escala de cinco pontos (1=certamente não compraria, 5=certamente compraria) (MEILGAARD et al., 1999).

Os resultados dos testes e da avaliação dos provadores foram tratados por análise de variância (ANOVA) e o Teste de Tukey foi usado para verificar diferenças estatísticas entre as amostras, ambos ao nível de 5\% de significância.

O projeto que deu origem ao presente trabalho foi submetido e aprovado por comitê de ética em maio de 2015, protocolo no Protocolo n. 42782614.7.0000.0106. 
As análises da composição centesimal foram feitas na polpa de abacaxi, na formulação de sorvete padrão e na formulação com polpa de abacaxi de melhor aceitação sensorial. Todas as análises foram feitas em triplicata.

O conteúdo de umidade foi determinado através do método gravimétrico, baseando-se na perda de peso do material submetido ao aquecimento em estufa (Marca Odontobras, Brasil) a $105^{\circ} \mathrm{C}$ até peso constante.

O teor de proteínas foi determinado através da avaliação do nitrogênio total da amostra, pelo método KJELDAHL (AACC 1995). Utilizou-se o fator de conversão de nitrogênio para proteína de 6,25.

O teor de lipídeos foi determinado pelo método de BLIGH e DYER, 1959.

O teor de cinzas foi determinado por incineração do material em mufla (Marca Quimis, Brasil) a $550{ }^{\circ} \mathrm{C}$ até peso constante, conforme metodologia de AOAC, 2000 e LEES, 1979.

A quantificação de fibra bruta foi determinada pelo método de extração em ebulição, após uma digestão ácida e outra alcalina. A primeira extração foi com solução de $\mathrm{H}_{2} \mathrm{SO}_{4}(1,25 \% \mathrm{p} / \mathrm{v})$ por 30 minutos, seguida de filtração e lavagem. A segunda extração foi com $\mathrm{NaOH}(1,25 \% \mathrm{p} / \mathrm{v})$ por mais 30 minutos, seguida por filtração e lavagem, após secagem a $100^{\circ} \mathrm{C}$ até peso constante. Descontou-se o teor de cinzas para o cálculo do teor de fibras (BRASIL, 1991).

A quantidade de carboidrato foi determinada por diferença, subtraindo-se de 100 os teores em porcentagem de umidade, proteína, cinza e lipídeo. $\mathrm{O}$ valor dos carboidratos inclui as fibras totais.

\section{Resultados e Discussão}

As notas médias obtidas nos testes de aceitação das formulações de sorvetes avaliando os atributos aparência, cor, textura, aroma, sabor e aceitação global, dos tratamentos padrão, com 10\%, 20\% e 30\% de polpa de abacaxi estão apresentadas na Tabela 2.

Tabela 2: Notas dos testes de aceitação da aparência, cor, textura, aroma, sabor e aceitação global dos sorvetes.

\begin{tabular}{ccccc}
\hline Atributos & FP & F1 & F2 & F3 \\
\hline Cor & $6,82 \pm 1,88^{\mathrm{b}}$ & $7,70 \pm 1,43^{\mathrm{a}}$ & $8,02 \pm 1,15^{\mathrm{a}}$ & $8,18 \pm 0,98^{\mathrm{a}}$ \\
Aroma & $6,94 \pm 1,88^{\mathrm{b}}$ & $7,86 \pm 1,06^{\mathrm{a}}$ & $7,82 \pm 1,25^{\mathrm{a}}$ & $8,02 \pm 1,10^{\mathrm{a}}$ \\
Textura & $7,38 \pm 1,71^{\mathrm{a}}$ & $7,94 \pm 1,09^{\mathrm{a}}$ & $7,84 \pm 1,05^{\mathrm{a}}$ & $7,72 \pm 1,03^{\mathrm{a}}$ \\
Aparência & $7,28 \pm 1,48^{\mathrm{b}}$ & $7,86 \pm 1,17^{\mathrm{ab}}$ & $7,92 \pm 1,15^{\mathrm{ab}}$ & $8,02 \pm 1,09^{\mathrm{a}}$ \\
Sabor & $6,88 \pm 1,75^{\mathrm{b}}$ & $7,84 \pm 1,16^{\mathrm{a}}$ & $7,54 \pm 1,52^{\mathrm{ab}}$ & $7,34 \pm 1,42^{\mathrm{ab}}$ \\
Aceitação global & $6,82 \pm 1,48^{\mathrm{b}}$ & $7,70 \pm 1,29^{\mathrm{a}}$ & $7,66 \pm 1,55^{\mathrm{a}}$ & $7,00 \pm 1,99^{\mathrm{ab}}$ \\
\hline
\end{tabular}

FP-Formulação padrãosem adição depolpa;F1-Formulaçãocom 10\% de polpa;F2-Formulaçãocom 20\% de polpa;F3-Formulação com $30 \%$ de polpa. Médias com letras iguais na mesma linha indicam não haver diferença significativa entre os resultados $(p \leq 0,05)$, pelo teste de Tukey.

Em relação a todos os atributos, as formulações de sorvete com adição de polpa de abacaxi foram bem-aceitas com médias entre 7 e 8 ("gostei moderadamente" e "gostei muito") no teste de escala hedônica. Já a formulação de sorvete padrão apresentou predominantemente notas entre 6 e 7 ("gostei ligeiramente e gostei moderadamente"), as quais foram inferiores as notas obtidas para todos os atributos das outras formulações com adição de fruta. 
Os sorvetes com $10 \%, 20 \%$ e $30 \%$ de polpa de abacaxi não apresentaram diferença significativa entre si a $(\mathrm{p}<0,05)$, em relação a todos os atributos avaliados. A amostra padrão apresentou diferença significativa $a(p<0,05)$ em relação à amostra com $10 \%$ de polpa de abacaxi, em todos os atributos avaliados, excetuando-se os atributos aparência e textura.

O produto elaborado com polpa de abacaxi apresentou índices de aceitação elevados, observados com relação aos atributos, portanto sugere que o sorvete com polpa de abacaxi, nas três formulações elaboradas, oferece boas perspectivas de consumo e consequentemente de comercialização.

As notas atribuídas pelos provadores para a intenção de compra dos sorvetes encontram-se na Tabela 3.

Tabela 3. Intenção de compra dos sorvetes formulados com polpa de abacaxi.

\begin{tabular}{ccc}
\hline Tratamento & Média & \% de Aprovação* $^{*}$ \\
\hline Padrão & $3,78 \pm 1,31^{\text {a }}$ & 56 \\
$10 \%$ FBM & $4,24 \pm 0,82^{\text {a }}$ & 80 \\
$20 \% \mathrm{FBM}$ & $4,02 \pm 1,16^{\mathrm{a}}$ & 78 \\
$30 \% \mathrm{FBM}$ & $3,84 \pm 1,46^{\mathrm{a}}$ & 58 \\
\hline
\end{tabular}

Tratamentos: Escala: 1 = certamente não compraria; 2 = possivelmente não compraria; 3 =talvezcomprasse; talveznão comprasse; 4 = possivelmente compraria; 5 =certamente compraria. ${ }^{*}$ Porcentagem dos provadores queatribuíram notas 4 e 5 . Médias com letras iguais na mesma coluna não diferem entre si ao nível de 5\% de significância.

As formulações de sorvete com $10 \%$ e 20 \% de polpa de abacaxi apresentaram intenção de compra média positiva (nota 4) pelos julgadores. Não houve diferença significativa entre estes tratamentos na intenção de compra a $(\mathrm{p}<0,05)$, evidenciada com a análise do percentual de aprovação. O sorvete com $10 \%$ de polpa de abacaxi apresentou aprovação de $80 \%$ dos julgadores.

Não houve diferença significativa entre todas as formulações de sorvetes investigadas com relação à intenção de compra a $(\mathrm{p}<0,05)$. As formulações com adição de polpa de abacaxi não apresentaram diferença significativa entre si a $(\mathrm{p}<0,05)$, em relação a todos os atributos sensoriais avaliados.

Ingestão Diária Recomendada (IDR) é a quantidade de proteína, vitaminas e minerais que deve ser consumida diariamente para atender às necessidades nutricionais da maior parte dos indivíduos e grupos de pessoas de uma população sadia (ANVISA, 2005). Considerando-se que a Ingestão Diária Recomendada para Adultos de proteína é de 50 gramas e de cálcio é $1000 \mathrm{mg}$ e que a formulação com maior teor de polpa de abacaxi (30\%) não seria fonte de fibra (fonte de fibra 2,5 gramas em uma porção de 60 gramas de sorvete ANVISA, 2012) a amostra com 10\% de polpa de abacaxi foi escolhida para as análises físico-químicas por ela conter a maior quantidade de leite sem lactose com o maior teor de proteína e cálcio.

\section{Tabela 4. Características físico-químicas da polpa de abacaxi, formulação padrão e formulação com $10 \%$ de polpa de abacaxi.}

\begin{tabular}{cccc}
\hline Componente (\%) & Polpa de abacaxi & FP (\%) & F1 (\%) \\
\hline Umidade & $93,42 \pm 1,27$ & $84,08 \pm 3,71$ & $89,64 \pm 0,81$ \\
Cinzas & $0,27 \pm 0,20$ & $0,43 \pm 0,11$ & $0,50 \pm 0,10$ \\
Lipídeos & $0,13 \pm 0,07$ & $3,68 \pm 1,33$ & $2,57 \pm 1,35$ \\
Fibras & $0,19 \pm 0,002$ & $0,02 \pm 0,004$ & $0,03 \pm 0,005$ \\
Carboidratos & $4,59 \pm 0,005$ & $8,44 \pm 0,02$ & $4,96 \pm 0,005$ \\
Proteínas & $0,47 \pm 0,07$ & $3,37 \pm 0,97$ & $2,34 \pm 0,38$ \\
\hline
\end{tabular}

FP - Formulação padrão sem adição de polpa; F1- Formulação com 10\% de polpa. 
O sorvete com 10\% de polpa de abacaxi apresentou um maior teor de cinzas e fibras e um menor teor de proteína e lipídeos em relação ao sorvete padrão. $\mathrm{O}$ fato de haver elevação em porcentagem de fibras e cinzas já é esperado devido à adição da polpa de abacaxi, que é rica nesses componentes. Conforme mencionado por Madrid (1996), os sorvetes por serem constituídos de leite e polpa de frutas, são ricos em minerais, como cálcio, sódio, potássio e magnésio entre outros.

Através da Tabela 4 pode-se observar que a quantidade de lipídios de 2,57\% na formulação com $10 \%$ de polpa de abacaxi obedece ao Regulamento Técnico referente a gelados comestíveis (BRASIL, 1999), que estabelece teor mínimo de gordura para o sorvete de leite em 2,5\%.

A Tabela Brasileira de Composição de Alimentos (TACO, 2011), traz a composição da polpa de abacaxi em \% de: umidade 91,3; cinzas 0,3; lipídeos 0,1; fibra alimentar 0,3 e proteínas 0,5. Portanto, os resultados apresentados neste trabalho para composição da polpa de abacaxi na Tabela 4 são muito próximos aos reportados na TACO (2011), indicando que a polpa de abacaxi apresenta parâmetros desejados de qualidade nutricional.

\section{Conclusão}

Os resultados permitem concluir que a adição de polpa de abacaxi como ingrediente no sorvete sem lactose melhorou as características organolépticas do produto. $\mathrm{O}$ sorvete com 10 $\%$ de polpa de abacaxi traz teor reduzido de lipídeos e carboidratos e maior teor de cinzas, em comparação a formulação padrão sem polpa de abacaxi e apresentou aprovação sensorial, portanto um produto com qualidade que traz os benefícios nutricionais ao consumidor. Os resultados deste trabalho podem contribuir para o desenvolvimento de novos produtos para a indústria alimentícia nas linhas de tendência saudabilidade e sensoriabilidade e prazer.

\section{Referências}

\section{AACC - AMERICAN ASSOCIATION OF CEREAL CHEMISTS. Approved methods. 9. ed. Saint Paul, 1995.}

ABIS - Associação Brasileira das Indústrias e do Setor de Sorvetes - História do Sorvete. Você sabia que esta delícia existe há mais de 3000 anos? Disponível em: < http:// www.abis.com.br/instucional_historia.html> Acesso em: 10 de Agosto de 2016.

ABNT. Associação Brasileira de Normas Técnicas. Métodos de análise sensorial de alimentos e bebidas: classificação. - NBR 12994. São Paulo: ABNT, 1994.

ANALYSIS OF ASSOCIATION OF OFFICIAL ANALYTICAL CHEMISTS AOAC. Official Methods of Analysis of Association of Official Analytical Chemists. 13 ed. Washington, DC, 2000.

ANVISA - Agência Nacional de Vigilância Sanitária. Resolução da Diretoria Colegiada RDC no 269. Regulamento técnico sobre a ingestão diária recomendada de proteínas, vitaminas e minerais. Publicada no Diário Oficial da União (DOU) no 184, de 23 de setembro de 2005 . 
ANVISA - Agência Nacional de Vigilância Sanitária. Resolução da Diretoria Colegiada RDC no 54. Regulamento técnico sobre informação nutricional complementar, de 12 de Novembro de 2012.

BRASIL. Portaria n. 108, de 04 de setembro de 1991. Normas gerais de amostragem para análise de rotina. Método número 11 - Fibra Bruta. Diário Oficial da República Federativa do Brasil, Brasília, p. 19813, 17 set. 1991. Seção 1.

BRASIL. Ministério de Estado da Agricultura, Pecuária e Abastecimento. Portaria n ${ }^{\circ}$ 379 de 26 de Abril de 1999. Diário Oficial da República Federativa do Brasil, Brasília, abr. 1999.

BLIGH, E.G.; DYER, W.J. A rapid method of total lipid extraction and purification. Can J Biochem Physiol., v.37, n.8, p.911-917, 1959.

DUTCOSKY, S.D. Análise sensorial de alimentos. 20.ed. Curitiba: Universitária Champagnat, 1996.123p.

FAEDO (2013) Apud DIETRICH,J. M. Intolerância a lactose, um nicho de mercado a ser explorado. Revista Leite 2011. Disponível em: <http://globalfood.com.br/site/site/ arquivos/Intolerancia\%20a\%20lactose\%20por\%20Jaime\%20\%20\%20Marcos\%20Dietrich. pdf $>$. Acesso em: 26.ago.2012.

LEES, R. Manual de análises de alimentos. Zaragoza: Acribia, 1979. 130p.

LONGO, G. Influência da adição de lactase na produção de iogurte. 2006. Dissertação (Mestrado em Tecnologia de Alimentos) - Setor de Tecnologia, Universidade Federal do Paraná, Curitiba, 2006.

MADRID, A. V.; CENZAND, I.; VICENTE, J. M. Manual de Indústrias dos Alimentos. Tradução José A. Ceselin. São Paulo, Varela, 1996. 559p.

MEILGAARD, M.; CIVILLE, G.V.; CARR, B.T. Sensory evaluation techniques.3.ed. New York:CRC, 1999. 281 p.

PEREDA, Juan A. Ordóñez (Org.). Tecnologia de alimentos: alimentos de origem animal. Porto Alegre: Artmed, 2007.v. 2. 279 p.

QUEIROZ, M.I.; TREPTOW, R.O.Análise sensorial para avaliação da qualidade dos alimentos. Rio Grande: FURG, 2006. 268 p.

SILVA, Silvestre. Frutas no Brasil. 1.ed. São Paulo: Empresa das Artes, 1996.

TACO. Tabela Brasileira de Composição de Alimentos/NEPA-UNICAMP.- 4. ed. Campinas: NEPA-UNICAMP, 2011. 